\title{
A NOTE ON ROMAN DOMINATION OF DIGRAPHS
}

\author{
Guoliang Hao, Zhinong Xie \\ College of Science \\ East China University of Technology \\ Nanchang 330013, P.R. China \\ e-mail: guoliang-hao@163.com \\ xiezh168@163.com \\ AND \\ XiaOdan CHEN $^{1}$ \\ College of Mathematics and Information Science \\ Guangxi University \\ Nanning 530004, P.R. China \\ e-mail: x.d.chen@live.cn
}

\begin{abstract}
A vertex subset $S$ of a digraph $D$ is called a dominating set of $D$ if every vertex not in $S$ is adjacent from at least one vertex in $S$. The domination number of a digraph $D$, denoted by $\gamma(D)$, is the minimum cardinality of a dominating set of $D$. A Roman dominating function (RDF) on a digraph $D$ is a function $f: V(D) \rightarrow\{0,1,2\}$ satisfying the condition that every vertex $v$ with $f(v)=0$ has an in-neighbor $u$ with $f(u)=2$. The weight of an RDF $f$ is the value $\omega(f)=\sum_{v \in V(D)} f(v)$. The Roman domination number of a digraph $D$, denoted by $\gamma_{R}(D)$, is the minimum weight of an RDF on $D$. In this paper, for any integer $k$ with $2 \leq k \leq \gamma(D)$, we characterize the digraphs $D$ of order $n \geq 4$ with $\delta^{-}(D) \geq 1$ for which $\gamma_{R}(D)=\gamma(D)+k$ holds. We also characterize the digraphs $D$ of order $n \geq k$ with $\gamma_{R}(D)=k$ for any positive integer $k$. In addition, we present a Nordhaus-Gaddum bound on the Roman domination number of digraphs.
\end{abstract}

Keywords: Roman domination number, domination number, digraph, Nordhaus-Gaddum.

2010 Mathematics Subject Classification: 05C69, 05C20.

\footnotetext{
${ }^{1}$ Corresponding author.
} 


\section{REFERENCES}

[1] J.D. Alvarado, S. Dantas and D. Rautenbach, Strong equality of Roman and weak Roman domination in trees, Discrete Appl. Math. 208 (2016) 19-26. doi:10.1016/j.dam.2016.03.004

[2] J.A. Bondy and U.S.R. Murty, Graph Theory (GTM 244, Springer, 2008).

[3] Y. Caro and M.A. Henning, Directed domination in oriented graphs, Discrete Appl. Math. 160 (2012) 1053-1063. doi:10.1016/j.dam.2011.12.027

[4] E.W. Chambers, B. Kinnersley, N. Prince and D.B. West, Extremal problems for Roman domination, SIAM J. Discrete Math. 23 (2009) 1575-1586. doi:10.1137/070699688

[5] M. Chellali and N.J. Rad, Strong equality between the Roman domination and independent Roman domination numbers in trees, Discuss. Math. Graph Theory 33 (2013) 337-346. doi:10.7151/dmgt.1669

[6] Y. Fu, Dominating set and converse dominating set of a directed graph, Amer. Math. Monthly 75 (1968) 861-863. doi: $10.2307 / 2314337$

[7] X. Fu, Y. Yang and B. Jiang, Roman domination in regular graphs, Discrete Math. 309 (2009) 1528-1537. doi:10.1016/j.disc.2008.03.006

[8] S. Gyürki, On the difference of the domination number of a digraph and of its reverse, Discrete Appl. Math. 160 (2012) 1270-1276. doi:10.1016/j.dam.2011.12.029

[9] G. Hao and J. Qian, On the sum of out-domination number and in-domination number of digraphs, Ars Combin. 119 (2015) 331-337.

[10] T.W. Haynes, S.T. Hedetniemi and P.J. Slater, Domination in Graphs: Advanced Topics (Marcel Dekker, Inc., New York, 1998).

[11] M. Kamaraj and P. Jakkammal, Directed Roman domination in digraphs, submitted.

[12] C.-H. Liu and G.J. Chang, Upper bounds on Roman domination numbers of graphs, Discrete Math. 312 (2012) 1386-1391. doi:10.1016/j.disc.2011.12.021

[13] C.-H. Liu and G.J. Chang, Roman domination on strongly chordal graphs, J. Comb. Optim. 26 (2013) 608-619. doi:10.1007/s10878-012-9482-y

[14] S.M. Sheikholeslami and L. Volkmann, The Roman domination number of a digraph, Acta Univ. Apulensis Math. Inform. 27 (2011) 77-86.

[15] S.M. Sheikholeslami and L. Volkmann, Signed Roman domination in digraphs, J. Comb. Optim. 30 (2015) 456-467. doi:10.1007/s10878-013-9648-2 
[16] T.K. Šumenjak, P. Pavlič and A. Tepeh, On the Roman domination in the lexicographic product of graphs, Discrete Appl. Math. 160 (2012) 2030-2036. doi:10.1016/j.dam.2012.04.008

[17] L. Volkmann, Signed total Roman domination in digraphs, Discuss. Math. Graph Theory 37 (2017) 261-272. doi:10.7151/dmgt.1929

[18] H.-M. Xing, X. Chen and X.-G. Chen, A note on Roman domination in graphs, Discrete Math. 306 (2006) 3338-3340.

doi:10.1016/j.disc.2006.06.018

Received 12 December 2016

Revised 23 March 2017

Accepted 23 March 2017 